\title{
Narrative Review: Bidirectional Association Between COVID-19 and Mental Health Disorders: ANarrative Review
}

\author{
Haleh Talaie $^{1^{*}}$ (D), Sayed Masoud Hosseini ${ }^{1}$ (D), Maryam Nazari' ${ }^{1}$ Farzad Nazemi²
}

1. Toxicological Research Center, Loghman-Hakim Hospital, Shahid Beheshti University of Medical Sciences, Tehran, Iran.

2. Psychiatrist, Loghman-Hakim Hospital Poison Center, Tehran, Iran.

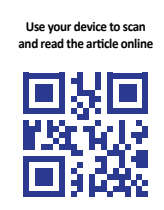

Chtat On: Talaie H, Hosseini SM, Nazari M, Nazemi F. Bidirectional Association Between COVID-19 and Mental Health Disorders: A Narrative Review. International Journal of Medical Toxicology and Forensic Medicine. 2021; 11(3):34101. https:// doi.org/10.32598/ijmtfm.v11i3.34104

dol ${ }^{\circ} h t$ tps://doi.org/10.32598/ijmtfm.v11i3.34104

\section{(i) (5)}

Article info:

Received: 14 Feb 2021

First Revision: 24 Mar 2021

Accepted: 3 Apr 2021

Published: 18 Jul 2021

\section{Keywords:}

Coronavirus Disease 2019

(COVID-19), Severe Acute

Respiratory Syndrome

Coronavirus 2 (SARS-CoV-2),

Mental disorders, Cytokine

release syndrome, Inflammation

\section{A B STRACT}

Background: Fear, anxiety, and stress are natural reactions of the human body to unpredictable and potentially threatening conditions. Currently, individuals are encountering Coronavirus Disease 2019 (COVID-19) pandemic; it is caused by a virulent, partially unknown pathogen with high transmissibility, recognized as Severe Acute Respiratory Syndrome Coronavirus 2 (SARS-CoV-2). It is conceivable to experience anxiety, stress, and subsequent mental health disorders during the pandemic. This narrative review provided a brief overview of mechanisms involved in COVID-19 and mental health disorders as well as the bidirectional association between them.

Methods: Scientific electronic databases, such as PubMed, Scopus, Web of Science, and Google Scholar were thoroughly searched, based on different keywords in this study.

Results: Lifestyle changes and isolation for breaking the chain of infection facilitate mental health disorders development more than before. Since the onset of the pandemic, the prevalence of mental health disorders has significantly increased. Moreover, studies suggested that the incidence of COVID-19 and the progression of the disease to a severe and life-threatening stage is higher in individuals with a history of mental health disorders. Inflammation is caused by, also may cause mental health disorders. Besides, COVID-19 is associated with cytokine storm and subsequent inflammation.

Conclusion: There seems to be a bidirectional association between COVID-19 and mental health disorders, in which inflammation acts as an adaptor. 


\section{Introduction}

thas been more than 10 months since CoronaviI rus Disease 2019 (COVID-19) was announced as a global health issue by the World Health Organization (WHO); however, no definite pharmacotherapy method has been developed in this regard [1,2]. As the Severe Acute Respiratory Syndrome Coronavirus 2 (SARS-CoV-2) spreads savagely, its associated high mortality and morbidity rates have led to great concern, worldwide [3, 4]. The severity of the disease and the mortality rate among the patients afflicted with COVID-19 depends on various factors, such as gender, age, underlying diseases, and even ethnicity [5-8]. Moreover, various definitions of COVID-19 cases exist in different countries. There might be difficulties in detecting positive cases and focusing more on symptomatic patients. Accordingly, it is not far from expectation to overestimate or underestimate the COVID-19 Case Fatality Ratio (CFR) [5]. Thus, in this stage, the naive estimation of the CFR of SARS-CoV-2 is hard to determine; however, the range of current estimates widely varies from $<0.1 \%$ to $>25 \%$ [5]. Moreover, SARS-CoV-2 has a high level of transmissibility and approximately $15 \%-20 \%$ of cases would develop the severe and life-threatening form of COVID-19 [9]. These characteristics of the COVID-19 pandemic along with socioeconomic concerns and prolonged isolation could result in triggering Mental Health Disorders (MHDs) $[10,11]$. Surveys revealed significant increases in the occurrence of MHDs worldwide. Based on the literature, the COVID-19 pandemic caused depressive symptoms in $16.5 \%-43.8 \%$ and anxiety disorders in $21.6 \%-45.1 \%$ of the studied populations [12]. Generally, it was reported that the prevalence of MHDs increased from $18.9 \%$ to $27.3 \%$ in Europe. Furthermore, the incidence of anxiety and depressive symptoms almost tripled in the USA in 2020, compared to 2019 [12].

It was suggested that inflammatory cytokines are causative factors in the pathophysiology of COVID-19 and MHDs development [13-15]. The present review aimed to illustrate the association between pre-existing or concurrent mental disorders and COVID-19 focusing on the involved mechanism and cellular signaling pathway. First, the intertwining of neuropsychiatric disorders with inflammation was demonstrated. Then, inflammatory reactions in COVID-19 were described. Finally, the bidirectional association between COVID-19 and MHDs as well as underlying inflammatory mechanisms were discussed.

\section{Materials and Methods}

The necessary data were collected from the published articles in PubMed, Scopus, Web of Science, and Google Scholar databases. For evaluating the association between COVID-19 and MHDs, we limited the publication date from 2020 to 2021. However, we have set no limitation on the date of publication to assess the involved mechanisms and the prevalence of MHDs in previous epidemics, such as Severe Acute Respiratory Syndrome (SARS) and Middle East Respiratory Syndrome (MERS). Moreover, the results of our published and conducted investigations were included. Related keywords and MeSH terms included the following: "COVID-19", "SARS-CoV-2", "mental disorders", "mental health", "depression", "anxiety", and "pandemic". After screening the records, 56 relevant studies were identified; from which, 35 articles met the inclusion criteria of the study. The inclusion criteria were published articles in English, matching between article's topic and research goals (the association between COVID-19 pandemic \& MHDs).

\section{MHDs and inflammatory cytokines}

MHDs (especially mood disorders) and inflammation are closely linked. Inflammation and MHDs, through a bidirectional association, potentiate each other [16]. Inflammation seems to be a precursor for MHDs [16, 17]; MHDs in turn, caused the activation of an inflammatory signaling pathway $[18,19]$. MHDs and inflammation crosstalk occur through different signaling pathways.

Numerous conditions could be considered as the precursor of MHDs. Some examples include anxiety, social anxiety disorder, chronic insomnia, childhood sexual abuse, and stroke [20-24]. These stressors, as sterile stimuli, resulting in the activation of inflammatory pathways via Damage-Associated Molecular Patterns (DAMPs) [16]. During tissue damage and cellular stress induced by mentioned stressors, DAMPs are released to activate repair mechanisms [25]. However, excessive DAMPs production is detrimental. Accordingly, studies revealed that DAMPs-induced chronic sterile inflammation may be associated with some MHDs, such as depression or Alzheimer's disease [25, 26]. The major DAMPs released from injured tissue are Heat Shock Proteins (HSPs), High Mobility Group Box 1 (HMGB1), S100b, extracellular ATP, and oxidized molecules $[16,26]$. These molecular patterns are sensed by Pattern Recognition Receptors (PRRs), expressed on various immune and nonimmune cells [27]. Toll-Like Receptors (TLRs) are a class of PRRs that recognize DAMPs and activate protective mechanisms. However, in the setting of pro- 
longed stress-related events, the post-translation process of TLRs signaling pathway is altered; this process may result in increased vulnerability to MHDs [28]. Different TLRs are involved in sterile inflammation consequent to prolonged stressors exposure. Some examples include cell surface TLRs, including TLR1,2,4,5,6, or endosomal TLRs, including TLR3,7,9 (Figure 1). Among them, TLR4 has been widely studied in MHDs [26]. TLR4 has two main signaling pathways; Myeloid differentiation primary response protein 88 (MyD88)-dependent pathway and MyD88-independent pathway. These signaling pathways end up with the entrance of transcription factors, such as Nuclear Factor $\kappa \mathrm{B}(\mathrm{NF}-\kappa \mathrm{B})$ and Interferon Regulatory Factor 3 (IRF3) into the nucleus; in turn, they regulate the expression of pro-inflammatory protein and cytokine, such as inducible Nitric Oxide Synthase (iNOS), Cyclooxygenase 2 (COX-2) enzyme, IL-1 $\beta$, IL-6, TNF- $\alpha$, IFN $\alpha$, IFN $\beta$, and IFN $\gamma$-inducible protein (Figure 1) $[28,29]$. It is well documented that elevated levels of inflammatory cytokine may be associated with increased risks of MHDs [26]. According to preclinical reports, knocking out of the TLR4 gene in mice results in less susceptibility to MHDs [30]. Moreover, the upregulation of TLR4 signaling pathway elements and final products (i.e., pro-inflammatory cytokine) were observed in patients with MHDs [31-34].

Long-Lasting exposure to stressor activates the stress system. This process leads to elevated plasma levels of Glucocorticoids (GCs), Norepinephrine (NP), and epinephrine. In healthy physiological conditions, exposure to acute stress results in cortisol secretion; subsequently, it binds to Glucocorticoid Receptors (GRs) and regulates the immune system and the Hypothalamic-PituitaryAdrenal (HPA) axis. Upon activation, GRs translocates into the nucleus and cause anti-inflammatory cytokine upregulation and inflammatory pathway inhibition. This is achieved by suppressing the pro-inflammatory cytokines transcription factors (i.e., NF- $\mathrm{BB}$ ). In contrast, chronic exposure to stressors leads to the constant production of cortisol. Chronic exposure to cortisol results in steroid resistance. Persistent TLRs signaling may decrease the capacity of the glucocorticoid receptor to inhibit NF- $\kappa \mathrm{B}$, which may contribute to glucocorticoid resistance (Figure 2) [35]. In other words, the frequency of GRs reduces in the setting of steroid resistance. GRs shortage results in enhanced inflammation and the risk of generating MHDs $[35,36]$. In a study, the resistance of lymphocytes to the inhibitory effects of dexamethasone in patients with Major Depressive Disorder (MDD) patients was reported in vitro [37]. A majority of studies on MDD revealed partial resistance to glucocorticoids and elevated pro-inflammatory cytokine [38].
Peripherally circulating cytokines may reach the Central Nervous System (CNS) via active cytokine transport across the Blood-Brain Barrier (BBB) (humoral route); they may also bind to receptors expressed on the afferent fiber, especially the vagus nerve, and affect the CNS (neural route) [39]. The metabolism and production of mood-regulating neurotransmitters, such as serotonin, glutamate, and dopamine may alter by an elevated level of pro-inflammatory cytokine. For example, inflammatory cytokines could alter tryptophan metabolism by activating Indoleamine 2,3-Dioxygenase (IDO). Tryptophan, as a serotonin precursor, is catabolized by IDO into kynurenine. Kynurenine in turn is metabolized into 3-Hydroxykynurenine (3-HK), Quinolinic Acid (QA), and Kynurenic Acid (KA). QA is an N-Methyl-D-Aspartate (NMDA) receptor agonist, KA is an NMDA receptor antagonist, and 3-HK is an oxidative stressor. In the setting of the elevated pro-inflammatory cytokine, the metabolism of kynurenine is in favor of QA production [40]. Thus, besides oxidative stress induced by 3-HK and the diminished production of serotonin due to the breaking down of tryptophan into kynurenine, QA overproduction and its agonistic effect on NMDA receptor; consequently, it results in increased glutamate release and decreased glutamate reuptake. Increased glutamate along with oxidative stress may lead to excitotoxicity and neurodegeneration and enhanced risk of MHDs (Figure 3) [41]. Previous studies suggested that depression-like behavior in mice could be disappeared by the inhibition of NMDA receptor or IDO activation [42, 43]. Moreover, Rasion et al. reported a strong correlation between increased Cerebrospinal Fluid (CSF) concentration of kynurenine and quinolinic acid and depressive symptoms in patients [44].

\section{COVID-19 and cytokine storm}

The exact pathology of COVID-19 remains unidentified. However, the immune-mediated injury seems to be the main causative event. It was suggested that Acute Respiratory Distress Syndrome (ARDS), as the main manifestation of COVID-19, is developed due to cytokine storm [45]. Different medical conditions may contribute to the development of cytokine storms, such as infection, rheumatic disease, malignant tumor, immunotherapeutic pharmacotherapy, and iatrogenic injury [44]. Among them, infection is the most common cause [46]. Cytokine storms might play a crucial role in SARS-CoV-2 pathogenesis [47, 48]. Furthermore, scholars indicated that cytokine storm is involved in the pathophysiology of other infectious diseases (e.g. influenza, SARS-CoV, MERS) [49-52]. 


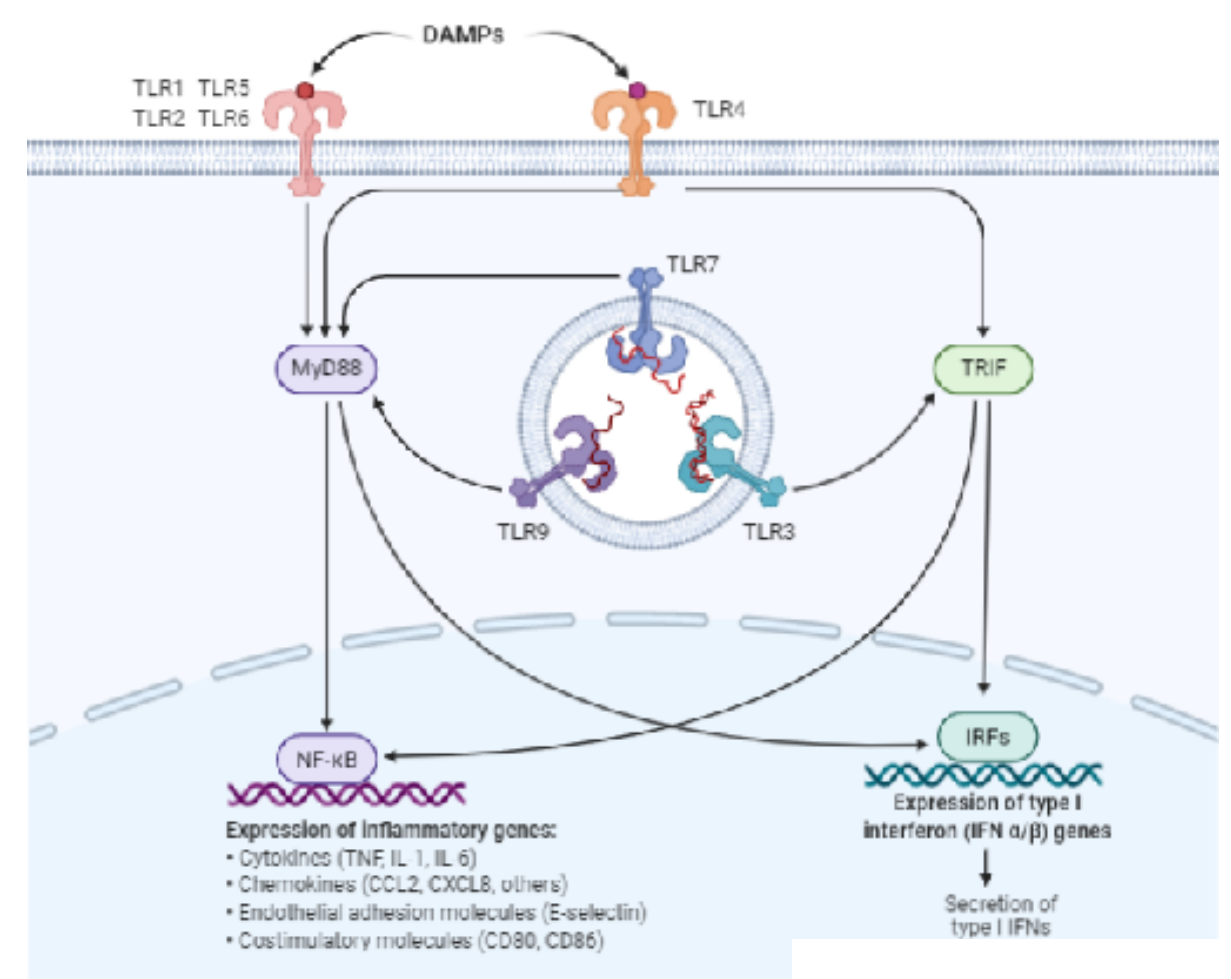

Figure 1. TLRs signaling pathway

DAMPs released from damaged tissue recognized by TLRs which result in increased inflammatory cytokine production. Cell surface TLRs (TLR1,2,4,5,6) or endosomal TLRs (TLR3,7,9) activation leads to transcription factors (NF-kB and IRF3) induction; in turn, it upregulates inflammatory markers expression.

DAMPs: Damage-associated molecular patterns; TLRs: Toll-like receptors; MyD88: Myeloid differentiation primary response 88; TRIF: TIR-domain-containing adapter-inducing interferon- $\beta$; NF-kB: Nuclear factor-kappa B; IRFs: Interferon regulatory factors (image created with BioRender.com).

Researchers have found that SARS-CoV-2 infection leads to cytokine storms through some conjecture mechanisms. Additionally, cytokine storms may be induced by the direct effect of SARS-CoV-2 [53]. The virus attaches to a specific receptor, called AngiotensinConverting Enzyme 2 (ACE2) through Spike (S) protein in nasal and bronchial epithelial cells and pneumocytes [54]. ACE2 is a single-pass type I membrane protein that converts vasoconstrictor angiotensin II to vasodilator angiotensin 1-7. The obtained data revealed that binding of the SARS-CoV-2 to its receptor (i.e., ACE2) results in ACE2 down-regulation; subsequently, it increases angiotensin II levels and decreases angiotensin 1-7 levels. Then, angiotensin II by binding to Angiotensin Receptor 1 (AT1R), plays its role as a pro-inflammatory cytokine. Accordingly, angiotensin II induces the activation of pro-inflammatory transcription factor NF- $\mathrm{kB}$ and STAT3 activation; through which measures, it regulates the expression of inflammatory cytokine resulting in a hyperinflammatory state and increased pulmonary vascular permeability (Figure 4) $[53,55,56]$.

Moreover, after the infection of pulmonary capillary cells, these cells promote immune system responses. This is achieved through the secretion of pro-inflammatory cytokines, such as IL-1, TNF- a, and IL-6 [57]. Infected alveolar capillary endothelial cells are targeted by the immune system. The number of $\mathrm{T}$ lymphocytes is also declined due to infection by the virus and subsequent apoptosis [58]. Studies revealed the reduction of $\mathrm{CD} 4+$ and $\mathrm{CD} 8+\mathrm{T}$ cells frequencies along with their over-activated status in COVID-19. This over-activation is characterized by a high concentration of cytotoxic granules in CD8+T cells and an increased number of inflammatory Th17 cells; eventually, this procedure results in severe immune injury in patients with COVID-19 [59, 60]. Furthermore, hype-inflammatory response of the immune system results in elevated production of Reac- 


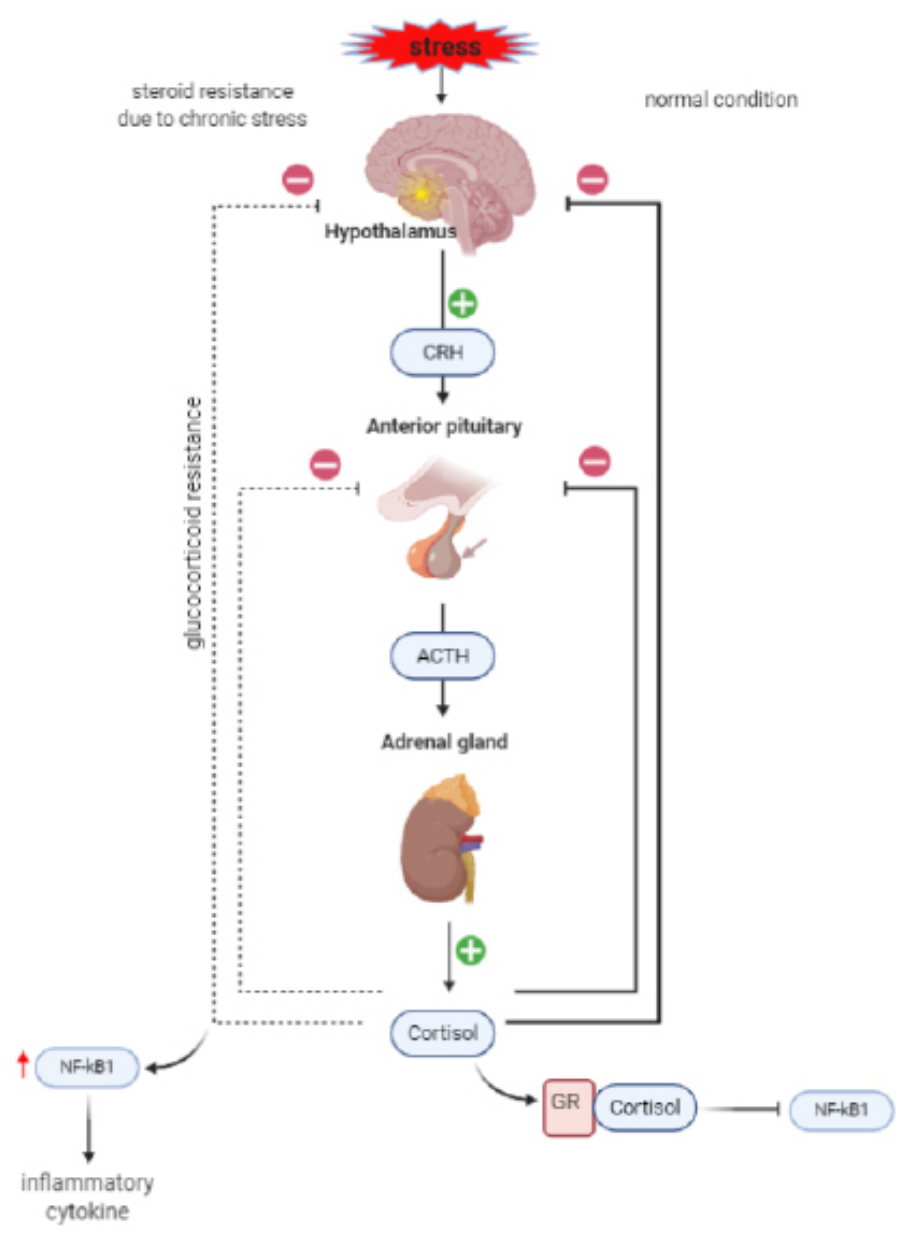

Figure 2. Hypothalamic-pituitary-adrenal axis dysregulation in chronic stress

Normally, stressor caused cortisol release which binds to GR and inhibits inflammation. Chronic stress caused glucocorticoid negative feedback attenuation and glucocorticoid resistance. In the setting of glucocorticoid resistance, the GRs decreased; thus, the inhibitory effect of cortisol on NF-kB was disrupted and caused an increase in the production of inflammatory cytokines.

CRH: Corticotropin-releasing hormone; Adrenocorticotropic hormone: Adrenocorticotropic hormone; GR: Glucocorticoid receptors; NF-kB: Nuclear factor-kappa B (image created with BioRender.com).

tive Oxygen Species (ROS) in infected epithelial cells. Increased ROS production is associated with the induction and activation of NF-кB; consequently, it upregulates the inflammatory cytokine expression [61].

High concentrations of circulating inflammatory cytokine derived from SARS-CoV-2 infection may disrupt the BBB [62]; thus, the cytokine network of the immune system and the cytokine network of the CNS crosstalk occurs. Peripheral inflammatory cytokine may cross through the disrupted BBB and activate microglia and astrocytes. Activated microglia and astrocytes can cause increased production of ROS and inflammatory cytokine in the CNS, leading to neuroinflammation. The CNS cy- tokine network activation, in turn, causes cytokine storm amplification (Figure 4) [61, 63].

\section{Bidirectional association between COVID-19 and MHDs}

MHDs are developed in a significant number of patients with COVID-19. Yanyu Hu et al. reported that a high frequency of patients infected with SARS-CoV-2 encountered insomnia (54.1\%), depression (45.9\%), and anxiety (38.8\%) [9]. Furthermore, a study conducted on 62354 patients with COVID-19 revealed that in the setting of SARS-CoV-2 infection, the risk of MHDs is considerably high, compared to influenza, or other respiratory tracts, or skin infections, urolithiasis, and cholelithiasis [64]. 


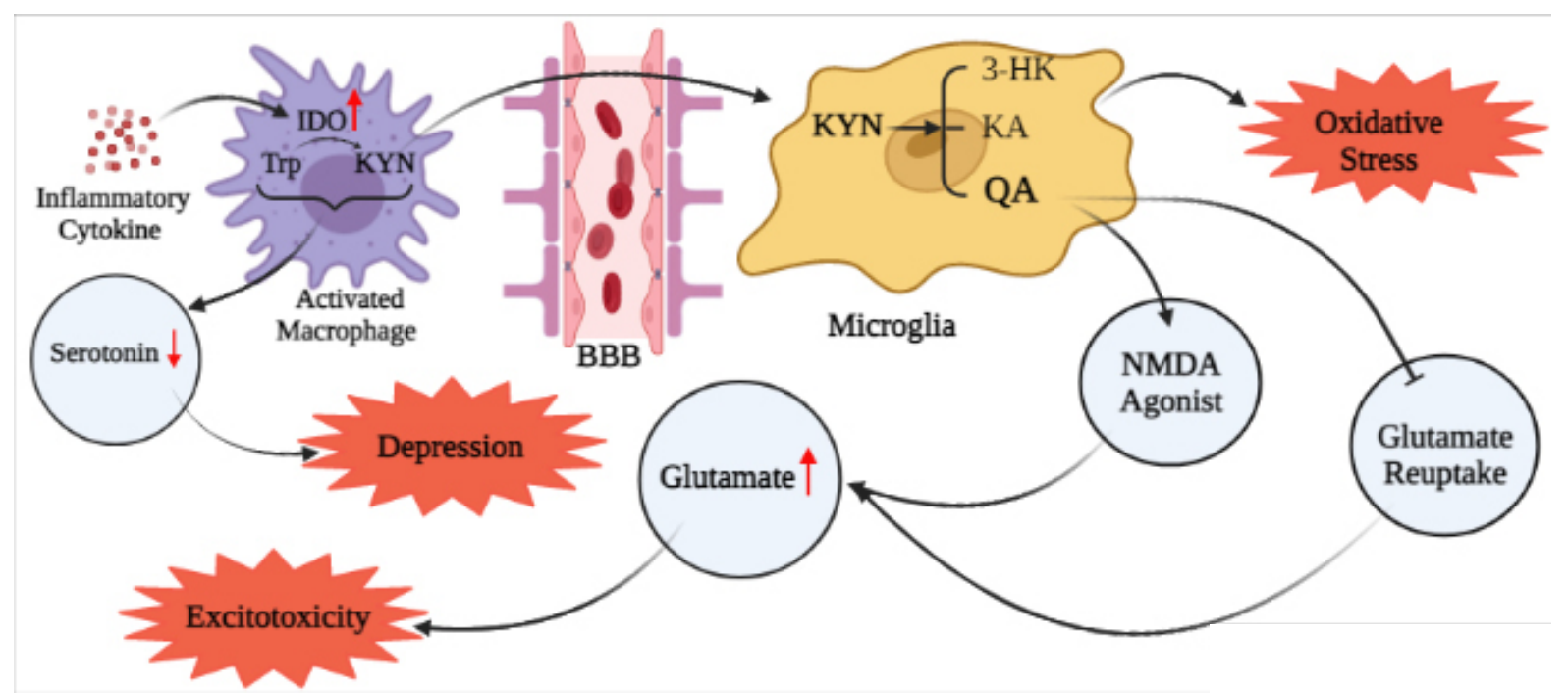

Figure 3. Inflammation-Induced excitotoxicity through the kynurenine pathway

Pro-inflammatory cytokines stimulate IDO in peripheral macrophages, resulting in the production of KYN, which crosses the blood-brain barrier. Microglia convert KYN to OA, KA, and 3-HK. In elevated pro-inflammatory cytokine conditions, the metabolism of kynurenine is in favor of QA production.3-HK is an oxidative stress inducer. QA exerts possible neurotoxic effects, by activating NMDA receptors and decreasing glutamate reuptake, which increases glutamate release to potentially excitotoxic levels. Besides, Trp metabolism toward serotonin production disrupts which triggers depression.

NMDA: N-methyl-D-aspartate; IDO: Indoleamine 2,3-dioxygenase (IDO); Trp: Tryptophan; KYN: Kynurenine; BBB: Bloodbrain barrier; QA: Quinolinic acid; 3-HK: 3-hydroxykynurenine; KA: Kynurenic acid (image created with BioRender.com).

The obtained data indicated that patients with a history of MHDs are more prone to SARS-CoV-2 infection. Moreover, the risk of COVID-19 infection is $65 \%$ more in patients with underlying MHDs, compared to mentally healthy subjects [64]. Wang et al. argued that the death rate in patients with COVID-19 and an underlying MHD (e.g. depression, schizophrenia) was higher than that of patients who were mentally healthy $(8.5 \%$ vs. $4.7 \%$ ). Besides, $27.4 \%$ of patients with COVID-19 and history of MHDs underwent hospitalization; however, only $18.6 \%$ of patients with COVID- 19 without mental health problems were hospitalized [65].

Therefore, in addition to the poor prognosis of mentally ill COVID-19 patients, the development of MHDs, also the augmentation of MHDs in patients with an underlying MHD were observed in the setting of COVID-19 infection. In addition to the socioeconomic impact of COVID-19 following prolonged quarantine, teleworking, permanent, or temporary unemployment and restriction in social activities $[66,67]$ may lead to the generation of MHDs. Accordingly, studies revealed that direct biological factors related to SARS-CoV-2 (e.g. consequent immune response, a load of the virus, or breathlessness) may play crucial roles in the aggravation or development of MHDs [14, 68, 69]. Thus, there seems to be a bidirectional association between COVID-19 and MHDs.
However, the exact mechanism accounting for this association remains undiscovered.

Cytokine storm is a situation of a sudden release of pro-inflammatory cytokines and interferon in a circulating system. This process could trigger the immigration of immune cells, such as macrophages, neutrophils, and $\mathrm{T}$ cells to the site of infection [60]. The invasive and dysregulated immune system reactions would destroy human organs (multi-organ failure) especially the lung [70]. Inflammatory markers infiltration and edema followed by ARDS in the late stages of the disease are the consequences of cytokine storm in pulmonary capillary cells; they would be lethal by decreasing the oxygen saturation levels [60]. Delirium, depression, anxiety, and insomnia are crucial mental health disturbances that may precipitate in some patients with COVID-19 [71]. Moreover, cytokine storm is involved in brain injuries and would prompt neuro-inflammation [72]. Additionally, these psychopathological hallmarks could be induced as a result of the viral infection of the CNS [73]. High-risk behaviors in mentally ill patients, including forgetting or rejecting to wear masks, not maintaining social distance, neglecting self-care or medical care, tobacco smoking, and substance abuse may put them at high risk for COVID-19 infection [74]. Some medications prescribed to patients with MHDs may increase the risk of coronavi- 


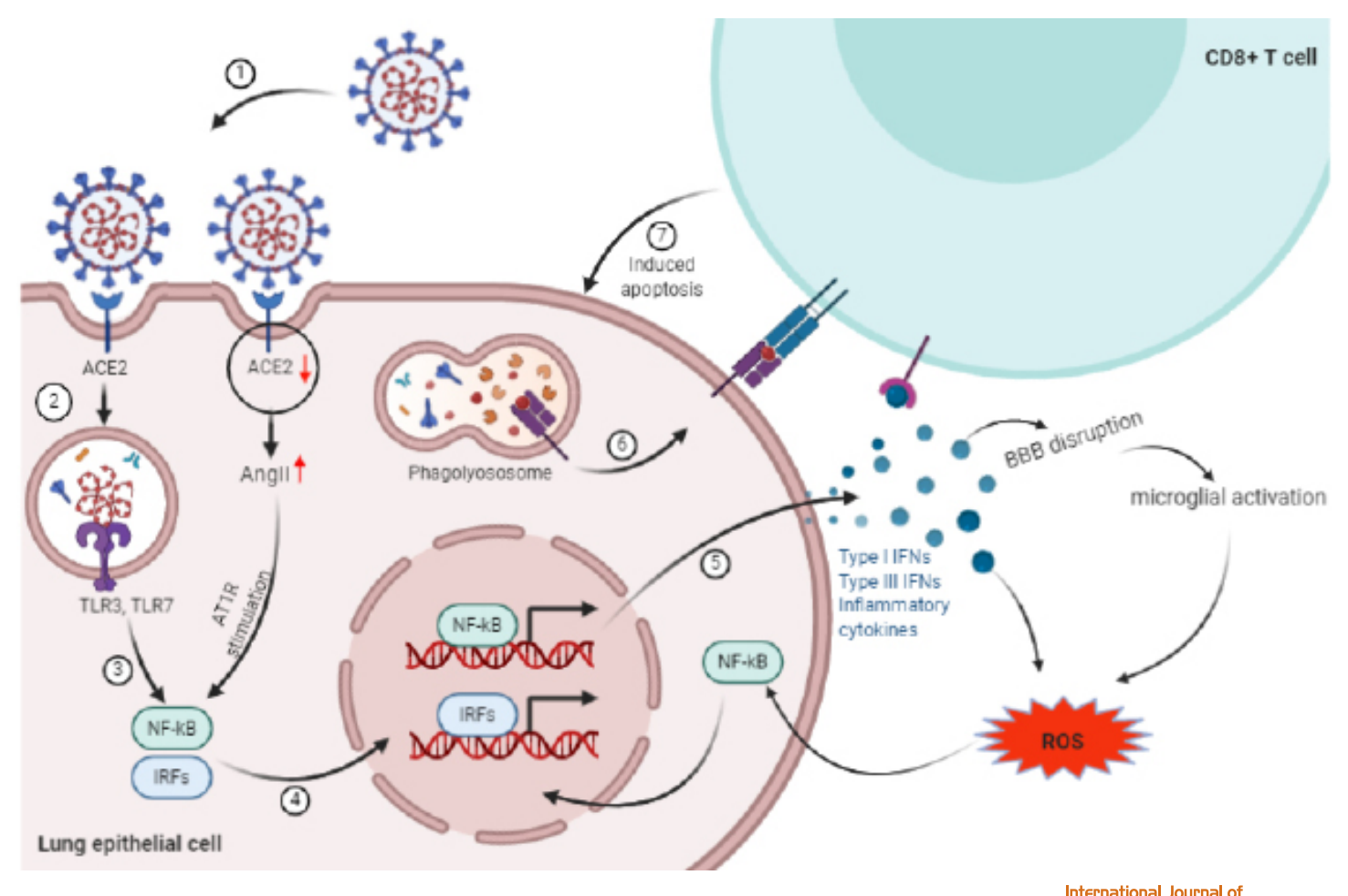

International Journal of
Medical Toxicology \& Forensic Medicine

Figure 4. Coronaviruses are RNA viruses, some of which can infect human lung epithelium via the receptor ACE2

Viral RNA activates endosomal TLR3,7. These receptors activate IRFs and NFkB to induce inflammatory cytokines. The antigen is presented on the surface of infected lung epithelial cells and CD8+ T cells induce apoptosis after the recognition of antigen on infected cells. Virus binding to ACE2 results in down-regulation of ACE2, which in turn caused increased levels of Ang II. Ang II by binding to AT1R plays its role as a pro-inflammatory cytokine. Inflammatory cytokine caused BBB disruption and microglial activation and finally ROS production.

ACE2: Angiotensin-converting enzyme 2; Ang: Angiotensin II; AT1R: angiotensin receptor 1; TLR: Toll-like receptor; NF-kB: Nuclear factor-kappa B; IRFs: Interferon regulatory factors; ROS: Reactive oxygen species; BBB: Blood-brain barrier (image created with BioRender.com).

rus infection. For instance, patients receiving Tricyclic or Tetracyclic Antidepressant (TCA/TeCA) presented a high level of C-reactive protein (CRP; an inflammatory biomarker) [75]. Moreover, benzodiazepines by activating GABAA receptors in immune cells cause susceptibility to infection [76].

Based on these findings and our previous study on inflammatory markers in patients with depression [77], inflammatory cytokines seem to be the major causative factor in the bidirectional association between COVID-19 and MHDs (Figure 5).

The Neutrophil to Lymphocyte Ratio (NLR) and the level of IL-1 $\beta$ in mentally ill COVID-19 patients was reported to be higher, compared to their mentally healthy counterparts [9]. Previous studies also demonstrated an increase in the levels of Interleukin (IL)-1 $\beta$, IL-4, IL-6, IL-10, CRP, Interferon (IFN)- $\gamma$, CXCL10, and CCL2 in COVID-19 $[49,78,79]$ as well as the levels of IL-1 $\beta$,
IL-6, IL-10, IFN- $\gamma$, TNF- $\alpha$, and transforming growth factor- $\beta$ (TGF- $\beta$ ) in MHDs [14, 80-81]. Interestingly, it was signified that the level of inflammatory markers, such as CRP in males with MHDs was higher than that in females; this difference may explain a higher vulnerability of men to COVID-19, compared to women [75, $82,83]$. As mentioned earlier, mechanisms involved in the pathophysiology of MHDs, including peripheral immune cell infiltration into the CNS, microglia activation, BBB disruption, HPA axis dysfunction, IDO induction, and neurotransmission impairment results in the upregulation of inflammatory cytokine. Besides, immune responses, in the setting of coronavirus infection leads to cytokine storm. Therefore, the coincidence of these conditions (i.e., coronavirus infection \& MHDs) may trigger the patient's therapeutic outcomes. 


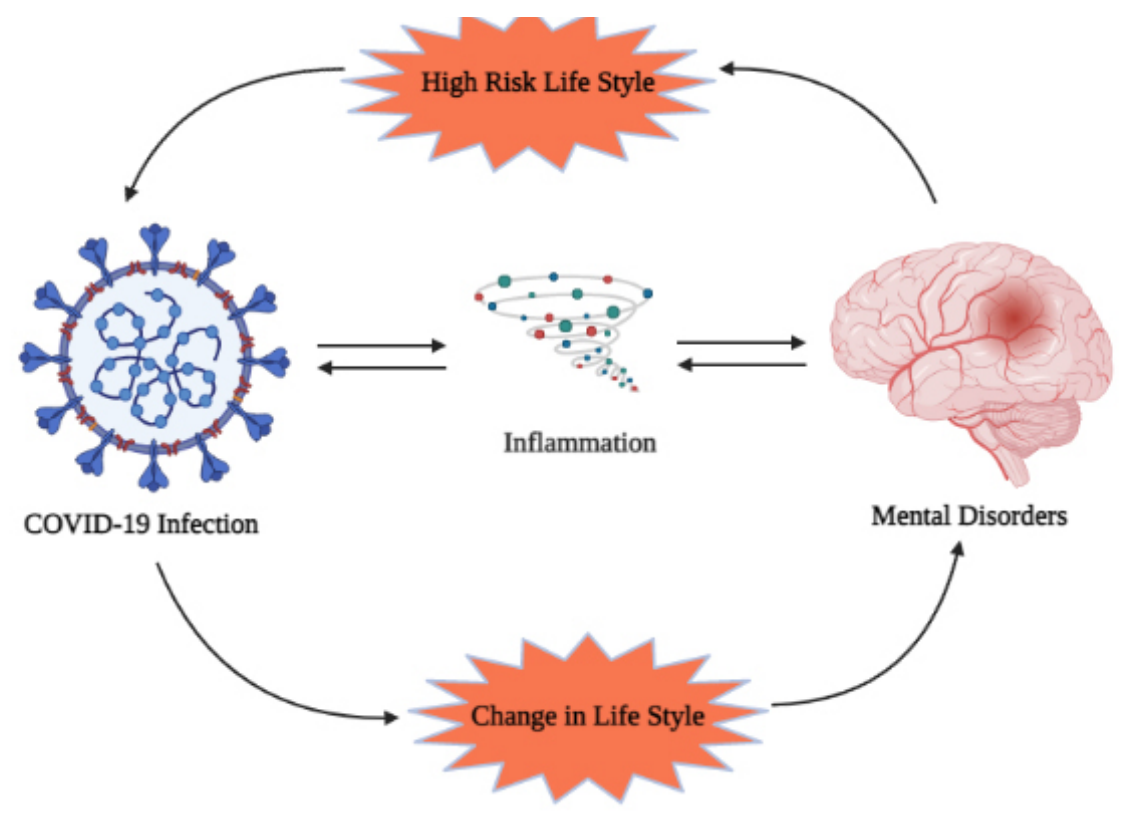

Figure 5. The bidirectional association of COVID-19 and MHDs International Journal of
Medical Toxicology \& forensic Medicine

COVID-19 results in an increased level of inflammatory cytokine (cytokine storm) which triggers or induces MHDs. MHDs caused an increased level of inflammatory cytokine, which in turn, facilitates or triggers COVID-19 through immune system dysfunction. The high-risk lifestyle of mentally ill individuals increases the odds of infection. Changes in lifestyle during pandemics (e.g. isolation or unemployment) increases the odds of developing MHDs (image created with BioRender.com).

\section{Conclusion}

Our findings indicated that the occurrence of pandemics significantly increased the prevalence of MHDs in society. There is also an elevated risk of developing COVID-19 in individuals with a history of MHDs. Therefore, providing the appropriate infrastructure to improve society members' mental health in the face of crises, like pandemics is critical. This measure requires adequate funding for community mental health care. Electronic mental health (e-mental health) is an online intervention to deliver mental health information, services, and care by which mental illnesses could be prevented and treated. The sustained evaluation of mental wellbeing, the early detection of vulnerable groups, and providing easy access to treatment for those with mental health issues would help reduce the incidence of MHDs in times of crisis, like pandemics. Modifying medications for individuals with MHDs under the guidance of a psychiatrist may also be considered. According to studies, patients receiving SSRI are less prone to SARS-CoV-2 infection, compared to patients receiving TCA or TeCA. Considering anti-inflammatory treatment with COX-2 inhibitors in patients with MHDs may also help minimize the risk of developing COVID-19.

\section{Ethical Considerations}

\section{Compliance with ethical guidelines}

There were no ethical considerations to be considered in this research.

\section{Funding}

This research received no grant from funding agencies in the public, commercial, or non-profit sectors.

\section{Author's contributions}

All authors equally contributed to the design and drafting of all research parts.

\section{Conflict of interest}

The authors declared no conflicts of interest.

\section{Acknowledgements}

We want to thank the Toxicological Research Center, Loghman Hakim Hospital, Shahid Beheshti University of Medical Sciences for their support. 


\section{References}

[1] Talaie H, Hosseini SM, Nazari M, Fakhri Y, Mousavizadeh A, Vatanpour H, et al. Is there any potential management against COVID-19? A systematic review and meta-analysis. Daru J Pharm Sci. 2020; 28(2):765-77. [DOI:10.1007/s40199020-00367-4] [PMID] [PMCID]

[2] Talaie H, Nazari M, Hosseini SM, Mousavizadeh A, AlaviDarazam I, Vatanpour H. The impact of current COVID-19 therapeutics on patients' clinical improvements based on disease severity; A systematic review. Int Pharm Acta. 2020; 3(1):e10. [DOI:10.22037/ipa.v3i1.32431]

[3] Merad M, Martin JC. Author correction: Pathological inflammation in patients with COVID-19: A key role for monocytes and macrophages. Nat Rev Immunol. 2020; 20(7):448. [DOI:10.1038/s41577-020-0353-y] [PMID] [PMCID]

[4] Blanco-Melo D, Nilsson-Payant BE, Liu WC, Uhl S, Hoagland $\mathrm{D}$, Møller R, et al. Imbalanced host response to SARS-CoV-2 drives development of COVID-19. Cell. 2020;181(5):1036-45.e9. [DOI:10.1016/j.cell.2020.04.026] [PMID] [PMCID]

[5] World Health Organization (WHO). Estimating mortality from COVID-19 [Internet]. 2020 [Updated 2020 August 4]. Available from: https://www.who.int/publications/i/ item/WHO-2019-nCoV-Sci-Brief-Mortality-2020.1

[6] Perez-Saez FJ, Lauer SA, Kaiser L, Regard S, Delaporte E, Guessous I, et al. Serology-informed estimates of SARS-COV-2 infection fatality risk in Geneva, Switzerland. Lancet Infect Dis. 2021; 21(4):e69-70. [DOI:10.1016/S1473-3099(20)30584-3] [PMID] [PMCID]

[7] Gold MS, Sehayek D, Gabrielli S, Zhang X, McCusker C, Ben-Shoshan M. COVID-19 and comorbidities: A systematic review and meta-analysis. Postgrad Med. 2020:1-7. [DOI:10.1 080/00325481.2020.1786964] [PMID]

[8] Jain V, Yuan JM. Predictive symptoms and comorbidities for severe COVID-19 and intensive care unit admission: A systematic review and meta-analysis. Int J Public Health. 2020; 65(5):533-46. [DOI:10.1007/s00038-020-01390-7] [PMID] [PMCID]

[9] Hu Y, Chen Y, Zheng Y, You C, Tan J, Hu L, et al. Factors related to mental health of inpatients with COVID-19 in Wuhan, China. Brain Behav Immun. 2020; 89:587-93. [DOI:10.1016/j. bbi.2020.07.016] [PMID] [PMCID]

[10] Asmundson GJG, Paluszek MM, Landry CA, Rachor GS, McKay D, Taylor S. Do pre-existing anxiety-related and mood disorders differentially impact COVID-19 stress responses and coping? J Anxiety Disord. 2020; 74:102271. [DOI:10.1016/j.janxdis.2020.102271] [PMID] [PMCID]

[11] Zandifar A, Badrfam R. Iranian mental health during the COVID-19 epidemic. Asian J Psychiatr. 2020; 51:101990. [DOI:10.1016/j.ajp.2020.101990] [PMID] [PMCID]

[12] Winkler P, Formanek T, Mlada K, Kagstrom A, Mohrova Z, Mohr $\mathrm{P}$, et al. Increase in prevalence of current mental disorders in the context of COVID-19: Analysis of repeated nationwide cross-sectional surveys. Epidemiol Psychiatr Sci. 2020; 29:e173. [DOI:10.1017/S2045796020000888] [PMID] [PMCID]

[13] Hao F, Tan W, Jiang L, Zhang L, Zhao X, Zou Y, et al. Do psychiatric patients experience more psychiatric symptoms during COVID-19 pandemic and lockdown? A case-control study with service and research implications for immunopsychiatry. Brain
Behav Immun. 2020; 87:100-6. [DOI:10.1016/j.bbi.2020.04.069] [PMID] [PMCID]

[14] Mazza MG, De Lorenzo R, Conte C, Poletti S, Vai B, Bollettini I, et al. Anxiety and depression in COVID-19 survivors: Role of inflammatory and clinical predictors. Brain Behav Immun. 2020; 89:594-600. [DOI:10.1016/j.bbi.2020.07.037] [PMID] [PMCID]

[15] Kempuraj D, Selvakumar GP, Ahmed ME, Raikwar SP, Thangavel R, Khan A, et al. COVID-19, mast cells, cytokine storm, psychological stress, and neuroinflammation. Neuroscientist. 2020; 26(5-6):402-14. [DOI:10.1177/1073858420941476] [PMID]

[16] Bauer ME, Teixeira AL. Inflammation in psychiatric disorders: What comes first? Ann N Y Acad Sci. 2019; 1437(1):57-67. [DOI:10.1111/nyas.13712] [PMID]

[17] Leonard BE. Inflammation and depression: A causal or coincidental link to the pathophysiology? Acta Neuropsychiatr. 2018; 30(1):1-16. [DOI:10.1017/neu.2016.69] [PMID]

[18] Hiles SA, Baker AL, de Malmanche T, McEvoy M, Boyle M, Attia J. The role of inflammatory markers in explaining the association between depression and cardiovascular hospitalisations. J Behav Med. 2015; 38(4):609-19. [DOI:10.1007/s10865-015-9637-2] [PMID]

[19] Masih J, Verbeke W. Immune system function and its relation to depression: How exercise can alter the immune system-depression dynamics. J Depress Anxiety. 2018; 7(4):1-7. [DOI:10.4172/2167-1044.1000325]

[20] Rice F, van den Bree MBM, Thapar A. A population-based study of anxiety as a precursor for depression in childhood and adolescence. BMC Psychiatry. 2004; 4(1):43. [DOI:10.1186/1471 244X-4-43] [PMID] [PMCID]

[21] Kraines MA, White EJ, Grant DM, Wells TT. Social anxiety as a precursor for depression: Influence of interpersonal rejection and attention to emotional stimuli. Psychiatry Res. 2019; 275:296-303. [DOI:10.1016/j.psychres.2019.04.001] [PMID]

[22] Baglioni C, Riemann D. Is chronic insomnia a precursor to major depression? Epidemiological and biological findings. Curr Psychiatry Rep. 2012;14(5):511-8. [DOI:10.1007/s11920-012-03085] [PMID]

[23] Boudewyn AC, Liem JH. Childhood sexual abuse as a precursor to depression and self-destructive behavior in adulthood. J Trauma Stress. 1995; 8(3):445-59. [DOI:10.1002/jts.2490080307] [PMID]

[24] Robinson RG, Jorge RE. Post-stroke depression: A review. Am J Psychiatry. 2016; 173(3):221-31. [DOI:10.1176/appi. ajp.2015.15030363] [PMID]

[25] Patel S. Danger-Associated Molecular Patterns (DAMPs): The derivatives and triggers of inflammation. Curr Allergy Asthma Rep. 2018; 18(11):63. [DOI:10.1007/s11882-018-0817-3] [PMID]

[26] Franklin TC, Xu C, Duman RS. Depression and sterile inflammation: Essential role of danger associated molecular patterns. Brain Behav Immun. 2018; 72:2-13. [DOI:10.1016/j. bbi.2017.10.025] [PMID]

[27] Santecchia I, Ferrer MF, Vieira ML, Gómez RM, Werts C. Phagocyte escape of leptospira: The role of TLRs and NLRs. Front Immunol. 2020; 11:571816. [DOI:10.3389/fimmu.2020.571816] [PMID] [PMCID] 
[28] Figueroa-Hall LK, Paulus MP, Savitz J. Toll-like receptor signaling in depression. Psychoneuroendocrinology. 2020 121:104843. [DOI:10.1016/j.psyneuen.2020.104843] [PMID]

[29] Bueno BG, Caso JR, Madrigal JLM, Leza JC. Innate immune receptor Toll-like receptor 4 signalling in neuropsychiatric diseases. Neurosci Biobehav Rev. 2016; 64:134-47. [DOI:10.1016/j. neubiorev.2016.02.013] [PMID]

[30] Cheng Y, Pardo M, de Souza Armini R, Martinez A, Mouhsine $\mathrm{H}$, Zagury JF, et al. Stress-induced neuroinflammation is mediated by GSK3-dependent TLR $_{4}$ signaling that promotes susceptibility to depression-like behavior. Brain Behav Immun. 2016; 53:207-22. [DOI:10.1016/j.bbi.2015.12.012] [PMID] [PMCID]

[31] Hajebrahimi B, Bagheri M, Hassanshahi G, Nazari M, Bidaki $\mathrm{R}$, Khodadadi $\mathrm{H}$, et al. The adapter proteins of TLRs, TRIF and MYD88, are upregulated in depressed individuals. Int J Psychiatry Clin Pract. 2014; 18(1):41-4. [DOI:10.3109/13651501.201 3.859708] [PMID]

[32] Dahl J, Ormstad H, Aass HCD, Malt UF, Bendz LT, Sandvik $\mathrm{L}$, et al. The plasma levels of various cytokines are increased during ongoing depression and are reduced to normal levels after recovery. Psychoneuroendocrinology. 2014; 45:77-86, [DOI:10.1016/j.psyneuen.2014.03.019] [PMID]

[33] Dowlati Y, Herrmann N, Swardfager W, Liu H, Sham L, Reim EK, et al. A meta-analysis of cytokines in major depression. Biol Psychiatry. 2010; 67(5):446-57. [DOI:10.1016/j.biopsych.2009.09.033] [PMID]

[34] Fan N, Luo Y, Ou Y, He H. Altered serum levels of TNF-a, IL-6, and IL-18 in depressive disorder patients. Hum Psychopharmacol. 2017; 32(4):e2588. [DOI:10.1002/hup.2588] [PMID]

[35] Cain DW, Cidlowski JA. Immune regulation by glucocorticoids. Nat Rev Immunol. 2017; 17(4):233-47. [DOI:10.1038/ nri.2017.1] [PMID]

[36] Heim C, Newport DJ, Mletzko T, Miller AH, Nemeroff CB. The link between childhood trauma and depression: Insights from HPA axis studies in humans. Psychoneuroendocrinology. 2008; 33(6):693-710. [DOI:10.1016/j.psyneuen.2008.03.008] [PMID]

[37] Bauer ME, Papadopoulos A, Poon L, Perks P, Lightman SL, Checkley S, et al. Altered glucocorticoid immunoregulation in treatment resistant depression. Psychoneuroendocrinology. 2003; 28(1):49-65. [DOI:10.1016/S0306-4530(02)00009-4] [PMID]

[38] Horowitz MA, Zunszain PA. Neuroimmune and neuroendocrine abnormalities in depression: Two sides of the same coin. Ann N Y Acad Sci. 2015; 1351(1):68-79. [DOI:10.1111/ nyas.12781] [PMID]

[39] Miller AH, Maletic V, Raison CL. Inflammation and its discontents: The role of cytokines in the pathophysiology of major depression. Biol Psychiatry. 2009; 65(9):732-41. [DOI:10.1016/j. biopsych.2008.11.029] [PMID] [PMCID]

[40] Hori H, Kim Y. Inflammation and post-traumatic stress disorder. Psychiatry Clin Neurosci. 2019; 73(4):143-53. [DOI:10.1111/ pcn.12820] [PMID]

[41] Miller AH, Raison CL. The role of inflammation in depression: From evolutionary imperative to modern treatment target. Nat Rev Immunol. 2016; 16(1):22-34. [DOI:10.1038/nri.2015.5] [PMID] [PMCID]
[42] Walker AK, Budac DP, Bisulco S, Lee AW, Smith RA, Beenders $B$, et al. NMDA receptor blockade by ketamine abrogates lipopolysaccharide-induced depressive-like behavior in C57BL/6J mice. Neuropsychopharmacology. 2013; 38(9):1609-16. [DOI:10.1038/ npp.2013.71] [PMID] [PMCID]

[43] O'Connor JC, Lawson MA, Andre C, Moreau M, Lestage J, Castanon N, et al. Lipopolysaccharide-induced depressive-like behavior is mediated by indoleamine 2, 3-dioxygenase activation in mice. Mol Psychiatry. 2009; 14(5):511-22. [DOI:10.1038/ sj.mp.4002148] [PMID] [PMCID]

[44] Raison CL, Dantzer R, Kelley KW, Lawson MA, Woolwine BJ, Vogt G, et al. CSF concentrations of brain tryptophan and kynurenines during immune stimulation with IFN-alpha: Relationship to CNS immune responses and depression. Mol Psychiatry. 2010; 15(4):393-403. [DOI:10.1038/mp.2009.116] [PMID] [PMCID]

[45] Shi Y, Wang G, Cai XP, Deng JW, Zheng L, Zhu HH, et al. An overview of COVID-19. J Zhejiang Univ Sci B. 2020; 21(5):343-60. [DOI:10.1631/jzus.B2000083] [PMID] [PMCID]

[46] Behrens EM, Koretzky GA. Review: Cytokine storm syndrome: Looking toward the precision medicine era. Arthritis Rheumatol. 2017; 69(6):1135-43. [DOI:10.1002/art.40071] [PMID]

[47] Chen N, Zhou M, Dong X, Qu J, Gong F, Han Y, et al. Epidemiological and clinical characteristics of 99 cases of 2019 novel coronavirus pneumonia in Wuhan, China: A descriptive study. Lancet. 2020; 395(10223):507-13. [DOI:10.1016/S01406736(20)30211-7] [PMID] [PMCID]

[48] Huang C, Wang Y, Li X, Ren L, Zhao J, Hu Y, et al. Clinical features of patients infected with 2019 novel coronavirus in Wuhan, China. Lancet. 2020; 395(10223):497-506. [DOI:10.1016/S01406736(20)30183-5]

[49] Channappanavar R, Perlman S. Pathogenic human coronavirus infections: Causes and consequences of cytokine storm and immunopathology. Semin Immunopathol. 2017; 39(5):529-39. [DOI:10.1007/s00281-017-0629-x] [PMID] [PMCID]

[50] Li Y, Chen M, Cao H, Zhu Y, Zheng J, Zhou H. Extraordinary GU-rich single-strand RNA identified from SARS coronavirus contributes an excessive innate immune response. Microbes Infect. 2013; 15(2):88-95. [DOI:10.1016/j.micinf.2012.10.008] [PMID] [PMCID]

[51] Lau SKP, Lau CCY, Chan KH, Li CPY, Chen H, Jin DY, et al. Delayed induction of proinflammatory cytokines and suppression of innate antiviral response by the novel Middle East respiratory syndrome coronavirus: Implications for pathogenesis and treatment. J Gen Virol. 2013; 94(Pt 12):2679-90. [DOI:10.1099/ vir.0.055533-0] [PMID]

[52] Oldstone MBA, Rosen H. Cytokine storm plays a direct role in the morbidity and mortality from influenza virus infection and is chemically treatable with a single sphingosine-1-phosphate agonist molecule. Curr Top Microbiol Immunol. 2014; 378:129-47. [DOI:10.1007/978-3-319-05879-5_6] [PMID] [PMCID]

[53] Gao YM, Xu G, Wang B, Liu BC. Cytokine storm syndrome in coronavirus disease 2019: A narrative review. J Intern Med. 2021; 289(2):147-61. [DOI:10.1111/joim.13144] [PMID] [PMCID]

[54] Hoffmann M, Kleine-Weber H, Schroeder S, Krüger N Herrler T, Erichsen S, et al. SARS-CoV-2 cell entry depends on ACE2 and TMPRSS2 and is blocked by a clinically proven protease inhibitor. Cell. 2020;181(2):271-80.e8. [DOI:10.1016/j. cell.2020.02.052] [PMID] [PMCID] 
[55] Eguchi S, Kawai T, Scalia R, Rizzo V. Understanding angiotensin II type 1 receptor signaling in vascular pathophysiology. Hypertension. 2018; 71(5):804-10. [DOI:10.1161/HYPERTENSIONAHA.118.10266] [PMID] [PMCID]

[56] Murakami M, Kamimura D, Hirano T. Pleiotropy and specificity: Insights from the interleukin 6 family of cytokines. Immunity. 2019; 50(4):812-31. [DOI:10.1016/j.immuni.2019.03.027] [PMID]

[57] Chen G, Wu D, Guo W, Cao Y, Huang D, Wang H, et al. Clinical and immunological features of severe and moderate coronavirus disease 2019. J Clin Invest. 2020; 130(5):2620-9. [DOI:10.1172/JCI137244] [PMID] [PMCID]

[58] Wong P, Pamer EG. CD8 T cell responses to infectious pathogens. Annu Rev Immunol. 2003; 21:29-70. [DOI:10.1146/annurev.immunol.21.120601.141114] [PMID]

[59] Bermejo-Martin JF, de Lejarazu RO, Pumarola T, Rello J, Almansa R, Ramírez P, et al. Th1 and Th17 hypercytokinemia as early host response signature in severe pandemic influenza. Crit Care. 2009; 13(6):R201. [DOI:10.1186/cc8208] [PMID] [PMCID]

[60] Xu Z, Shi L, Wang Y, Zhang J, Huang L, Zhang C, et al. Pathological findings of COVID-19 associated with acute respiratory distress syndrome. Lancet Respir Med. 2020; 8(4):420-2 [DOI:10.1016/S2213-2600(20)30076-X] [PMID] [PMCID]

[61] Bhaskar S, Sinha A, Banach M, Mittoo S, Weissert R, Kass JS, et al. Cytokine storm in COVID-19-immunopathological mechanisms, clinical considerations, and therapeutic approaches: The REPROGRAM consortium position paper. Front Immunol. 2020; 11:1648. [DOI:10.3389/fimmu.2020.01648] [PMID] [PMCID]

[62] Alexopoulos H, Magira E, Bitzogli K, Kafasi N, Vlachoyiannopoulos $\mathrm{P}$, Tzioufas A, et al. Anti-SARS-CoV-2 antibodies in the CSF, blood-brain barrier dysfunction, and neurological outcome: Studies in 8 stuporous and comatose patients. Neurol Neuroimmunol Neuroinflamm. 2020; 7(6):e893. [DOI:10.1212/NXI.0000000000000893] [PMID] [PMCID]

[63] Liu Q, Zhou YH, Yang ZQ. The cytokine storm of severe influenza and development of immunomodulatory therapy. Cell Mol Immunol. 2016; 13(1):3-10. [DOI:10.1038/ cmi.2015.74] [PMID] [PMCID]

[64] Taquet M, Luciano S, Geddes JR, Harrison PJ. Bidirectional associations between COVID-19 and psychiatric disorder: Retrospective cohort studies of 62354 COVID-19 cases in the USA. Lancet Psychiatry. 2021; 8(2):130-40. [DOI: 10.1016/ S2215-0366(20)30462-4]

[65] Seminog OO, Goldacre MJ. Risk of pneumonia and pneumococcal disease in people with severe mental illness: English record linkage studies. Thorax. 2013; 68(2):171-6. [DOI:10.1136/thoraxjnl-2012-202480] [PMID]

[66] Fana M, Pérez ST, Fernández-Macías E. Employment impact of Covid-19 crisis: From short term effects to long terms prospects. J Ind Bus Econ. 2020; 47:391-410. [DOI:10.1007/s40812020-00168-5] [PMCID]

[67] Poudel K, Subedi P. Impact of COVID-19 pandemic on socioeconomic and mental health aspects in Nepal. Int J Soc Psychiatry. 2020; 66(8):748-55. [DOI:10.1177/0020764020942247] [PMID] [PMCID]
[68] Postolache TT, Benros ME, Brenner LA. Targetable biological mechanisms implicated in emergent psychiatric conditions associated with SARS-CoV-2 infection. JAMA Psychiatry. 2021; 78(4):353-4. [DOI:10.1001/jamapsychiatry.2020.2795] [PMID]

[69] Gupta A, Madhavan MV, Sehgal K, Nair N, Mahajan S, Sehrawat TS, et al. Extrapulmonary manifestations of COVID-19. Nat Med. 2020; 26(7):1017-32. [DOI:10.1038/s41591-020-0968-3] [PMID]

[70] Gao Y, Li T, Han M, Li X, Wu D, Xu Y, et al. Diagnostic utility of clinical laboratory data determinations for patients with the severe COVID-19. J Med Virol. 2020; 92(7):791-6 [DOI:10.1002/ jmv.25770] [PMID] [PMCID]

[71] Rogers JP, Chesney E, Oliver D, Pollak TA, McGuire P, FusarPoli $\mathrm{P}$, et al. Psychiatric and neuropsychiatric presentations associated with severe coronavirus infections: A systematic review and meta-analysis with comparison to the COVID-19 pandemic. Lancet Psychiatry. 2020; 7(7):611-27. [DOI:10.1016/ S2215-0366(20)30203-0] [PMID] [PMCID]

[72] Dantzer R. Neuroimmune interactions: From the brain to the immune system and vice versa. Physiol Rev. 2018; 98(1):477-504. [DOI:10.1152/physrev.00039.2016] [PMID] [PMCID]

[73] Troyer EA, Kohn JN, Hong S. Are we facing a crashing wave of neuropsychiatric sequelae of COVID-19? Neuropsychiatric symptoms and potential immunologic mechanisms. Brain Behav Immun. 2020; 87:34-9. [DOI:10.1016/j.bbi.2020.04.027] [PMID] [PMCID]

[74] Wang QQ, Xu R, Volkow ND. Increased risk of COVID-19 infection and mortality in people with mental disorders: Analysis from electronic health records in the United States. World Psychiatry. 2021; 20(1):124-30. [DOI:10.1002/wps.20806] [PMID] [PMCID]

[75] Vogelzangs N, Beekman ATF, de Jonge P, Penninx BWJH. Anxiety disorders and inflammation in a large adult cohort. Transl Psychiatry. 2013; 3(4):e249. [DOI:10.1038/tp.2013.27] [PMID] [PMCID]

[76] Obiora E, Hubbard R, Sanders RD, Myles PR. The impact of benzodiazepines on occurrence of pneumonia and mortality from pneumonia: A nested case-control and survival analysis in a population-based cohort. Thorax. 2013; 68(2):163-70. [DOI:10.1136/thoraxjnl-2012-202374] [PMID]

[77] Talaie H, Abdollahi M, Pajoumand A, Panahandeh R, Barari $\mathrm{B}$, Baeeri M. Alteration of serum levels of interlukin 1 and tumor necrosis factor in depression independent of treatment or overdose of tricyclic antidepressants. Iran J Toxicol. 2009; 2(3):228-33 http:/ ijt.arakmu.ac.ir/article-1-73-en.html

[78] Ye Q, Wang B, Mao J. The pathogenesis and treatment of the 'Cytokine Storm' in COVID-19. J Infect. 2020; 80(6):607-13. [DOI:10.1016/j.jinf.2020.03.037] [PMID] [PMCID]

[79] Köhler CA, Freitas TH, Maes M, de Andrade NQ, Liu CS, Fernandes BS, et al. Peripheral cytokine and chemokine alterations in depression: A meta-analysis of 82 studies. Acta Psychiatr Scand. 2017; 135(5):373-87. [DOI:10.1111/acps.12698] [PMID]

[80] Renna ME, O'Toole MS, Spaeth PE, Lekander M, Mennin DS. The association between anxiety, traumatic stress, and obsessive-compulsive disorders and chronic inflammation: A systematic review and meta-analysis. Depress Anxiety. 2018; 35(11):1081-94. [DOI:10.1002/da.22790] [PMID] 
[81] Poletti S, Leone G, Hoogenboezem TA, Ghiglino D, Vai $\mathrm{B}$, de Wit $\mathrm{H}$, et al. Markers of neuroinflammation influence measures of cortical thickness in bipolar depression. Psychiatry Res Neuroimaging. 2019; 285:64-6. [DOI:10.1016/j.pscychresns.2019.01.009] [PMID]

[82] Liukkonen T, Räsänen $P$, Jokelainen J, Leinonen M, Järvelin MR, Meyer-Rochow VB, et al. The association between anxiety and C-Reactive Protein (CRP) levels: Results from the Northern Finland 1966 birth cohort study. Eur Psychiatry 2011; 26(6):363-9. [DOI:10.1016/j.eurpsy.2011.02.001] [PMID]

[83] Jin JM, Bai P, He W, Wu F, Liu XF, Han DM, =et al. Gender differences in patients with COVID-19: Focus on severity and mortality. Front Public Health. 2020; 8:152. [DOI:10.3389/ fpubh.2020.00152] [PMID] [PMCID] 